\title{
Determination of the yield function and its derivatives of the Barcelona Expansive model
}

\author{
Jamila El Brahmi ${ }^{1}$ et Nouzha Lamdouar ${ }^{1}$ \\ ${ }^{1}$ Ecole Mohammadia d'Ingénieurs, Rabat, Maroc
}

\begin{abstract}
The Barcelona Expansive Model "BE $\times$ M" presented by Alonso and al. in 1999 is one of the best models to correctly describe the hydromechanical behavior of an unsaturated expansive soil in the elastoplastic domain. The implementation of the $\mathrm{BE} \times \mathrm{M}$ in a computer code is, however, complicated by the existence of three yield surfaces (SI, SD and LC) that limit the elastic domain. In order to circumvent this difficulty, the yield surface " $F^{\prime}$ in the space (p*, q, s) is assimilated to a succession of ellipses along the suction axis "s" inclined by $45^{\circ}$ with respect to the two axes ( $\left.\mathrm{p}^{*}, \mathrm{~s}\right)$. Then, the equation of the yield surface corresponds to a given stress state. The partial derivatives of the yield function (with respect to the stress vector « $\sigma »$, the soil suction $« s »$ and the hardening parameter $\left\langle\mathrm{p}_{0}{ }^{*} »\right)$ are also determined since they make it possible to calculate the initial stresses $\dot{\sigma}_{i j}^{0}$ and $S_{i j}$ in the boundary integral equations of the expansive soil displacement.
\end{abstract}

Résumé. Le modèle de Barcelone «BE×M » présenté par Alonso et al. en 1999 est l'un des meilleurs modèles qui permettent de décrire correctement le comportement hydromécanique d'un sol gonflant non saturé dans le domaine élastoplastique. L'implémentation de BE $\times \mathrm{M}$ dans un code de calcul se trouve, néanmoins, compliquée par l'existence de trois surfaces de charge (SI, SD and LC) qui limitent le domaine élastique . Afin de contourner cette difficulté, la surface de charge « $\mathrm{F} »$ dans l'espace $\left(\mathrm{p}^{*}, \mathrm{q}, \mathrm{s}\right)$ est assimilée à une succession d'ellipses suivant l'axe des succions « $\mathrm{s} »$ inclinées de $45^{\circ}$ par rapport aux deux axes (p*, s). L'équation de la surface de charge correspond alors à un état de contrainte donné. Les dérivées partielles de la fonction de charge (par rapport au vecteur contrainte « $\sigma$ », à la succion « $S$ » et au paramètre d'écrouissage « $\left.\mathrm{p}_{0}{ }^{*} »\right)$ sont également déterminés puisqu'ils permettent de calculer les contraintes initiales $\dot{\sigma}_{i j}^{0}$ et $S_{i j}$ des équations d'intégrales de frontière du déplacement du sol gonflant.

\section{Introduction}

Dans le cadre de la modélisation élatoplastiques du comportement hydromécanique d'un sol gonflant non saturé avec le modèle $\mathrm{BE} \times \mathrm{M}$, nous sommes ramenés à résoudre les équations d'intégrale de frontière du déplacement suivants [1]

$$
\begin{aligned}
& \dot{U}_{j}(\xi)=\int_{S}\left[\dot{t}_{i}(x) G_{i j}(x, \xi)-F_{i j}(x, \xi) \dot{U}_{i}(x)\right] d S \\
& +\int_{V} \mathrm{~T}(1+2 \ln r) y_{j} \nabla^{2}(\dot{s}) d V+ \\
& \int_{S} \mathrm{~T}\left(\frac{2}{r^{2}} y_{j} y_{m} n_{m}+(1+2 \ln r) n_{j}\right) \dot{s} d S \\
& -\int_{S} \mathrm{~T}(1+2 \ln r) y_{j} \frac{\partial \dot{s}}{\partial n} d S+ \\
& \int_{V} \frac{\partial G_{i j}\left(x, \xi_{0}\right)}{\partial x_{k}}\left(\dot{\sigma}_{i k}^{0}-S_{i k} d s\right) d V
\end{aligned}
$$

avec:

$\xi$ et $x$ : les coordonnées du point test et du point source respectivement.

$\dot{t}_{i}$ et $\dot{U}_{i}$ : Incréments de traction et de déplacement sur la frontière du domaine $\mathrm{S}$.

$G_{i j}$ et $F_{i j}$ : Fonctions de Green (solutions singulières). $\nabla^{2}(\dot{s}), \dot{s}$ et $\frac{\partial \dot{s}}{\partial n}:$ Laplacien de la succion, les incréments de succion et de gradient de succion.

$r$ : distance entre le point test et le point source donnée par: $r^{2}=y_{i} y_{i}=\left(x_{1}-\xi_{1}\right)^{2}+\left(x_{2}-\xi_{2}\right)^{2}$ où $y_{i}=x_{i}-\xi_{i}$ 
$\mathrm{T}$ : un scalaire donné par : $\Gamma=\left(\lambda+\frac{2}{3} \mu\right) \frac{\kappa_{s}}{1+e} \cdot \frac{1}{s+p_{\text {atm }}}$

où: $\lambda, \mu:$ les constantes de Lamé

$\kappa_{s}$ : coefficient de compressibilité pour des incréments de succion dans le domaine élastique

$e:$ Indice des vides

$p_{\text {atm }}:$ pression atmosphérique

$S$ et $V:$ surface de frontière et domaine d'intégrales

$\dot{\sigma}_{i k}^{0}-S_{i k} d s$ : Contraintes initiales (contraintes plastiques) données par :

$$
\begin{aligned}
\dot{\sigma}_{i j}^{0}= & \frac{\left(\frac{\partial F}{\partial \sigma_{i j}}\right)^{T}\left(\lambda \delta_{i j} \dot{\varepsilon}_{k k}+2 \mu \dot{\varepsilon}_{i j}\right)\left(\lambda \delta_{i j} \frac{\partial F}{\partial \sigma_{k k}}+2 \mu \frac{\partial F}{\partial \sigma_{i j}}\right)}{H+\left(\frac{\partial F}{\partial \sigma_{i j}}\right)^{T}\left(\lambda \delta_{i j} \frac{\partial F}{\partial \sigma_{k k}}+2 \mu \frac{\partial F}{\partial \sigma_{i j}}\right)} \\
S_{i j}= & \left(\frac{\left(\left(\frac{\partial F}{\partial \sigma_{i j}}\right)^{T} \Gamma \delta_{i j}+\frac{\partial F}{\partial s}\right)\left(\lambda \delta_{i j} \frac{\partial F}{\partial \sigma_{k k}}+2 \mu \frac{\partial F}{\partial \sigma_{i j}}\right)}{H+\left(\frac{\partial F}{\partial \sigma_{i j}}\right)^{T}\left(\lambda \delta_{i j} \frac{\partial F}{\partial \sigma_{k k}}+2 \mu \frac{\partial F}{\partial \sigma_{i j}}\right)}\right)
\end{aligned}
$$

A condition d'avoir toutes les conditions aux limites requises, toutes les intégrales de la formule de déplacement peuvent êtres déterminées numériquement avec la méthode des éléments de frontière, sauf pour le dernier intégral volumique $\int_{V} \frac{\partial G_{i j}\left(x, \xi_{0}\right)}{\partial x_{k}}\left(\dot{\sigma}_{i k}^{0}-S_{i k} d s\right) d V$ qui nécessite un traitement particulier. Cette intégrale nécessite d'abord la détermination des contraintes plastiques du problème $\dot{\sigma}_{i k}^{0}-S_{i k} d s$ (appelées contraintes initiales) avant de calculer l'intégrale elle-même (qui n'est pas sans complications d'ailleurs).

Le calcul de $\dot{\sigma}_{i k}^{0}-S_{i k} d s$ repose sur la détermination de la fonction de charge $\langle\mathrm{F} »$ et de ses dérivées, d'où le présent article.

\section{Surfaces de charge du modèle $B E \times M$ :}

Le modèle de Barcelone « $\mathbf{B E} \times \mathbf{M}$ Model » $[2, \underline{3}]$ utilise, constitutivement, la contrainte nette $p^{*}=\frac{\sigma_{1}+\sigma_{2}+\sigma_{3}}{3}-u_{a}$ et la succion matricielle $s=u_{a}-u_{w}$ comme variables indépendantes pour décrire le comportement volumique des sols gonflants non saturés. Dans le cas anisotrope (triaxial), une troisième variable (contrainte déviatorique) définie par $q=\sigma_{1}-\sigma_{3}$ s'ajoute aux variables précédentes. Dans le cas général, le comportement du sol est décrit dans le plan $\left(p^{*}, \mathrm{q}, \mathrm{s}\right)$.

\subsection{Surface de charge « LC » dans le plan $\left(p^{*}, \mathrm{~s}\right)$}

Lors d'un essai de compression à succion contrôlée, on remarque qu'à une succion donnée $S$, la courbe de compressibilité est formée, dans le plan $\left(\mathrm{v}-\ln p^{*}\right)$, de deux droites caractérisées par les pentes $\kappa$ et $\lambda(s)$ [figure 1]. $\kappa$ étant le coefficient de compressibilité dans le domaine élastique et $\lambda(\mathrm{s})$ la pente de la partie normalement consolidée appelée aussi coefficient de compressibilité plastique [4]. Le coefficient $\kappa$ étant supposé constant, $\lambda(\mathrm{s})$ diminue quand la succion augmente (le sol tend à se rigidifier et sa compressibilité diminue). Ce dernier paramètre est modélisé par Alonso et al. [4] par l'expression suivante : $\lambda(s)=\lambda(0)\left[(1-r) e^{-\beta . s}+r\right]$

Avec :

$\lambda(0)$ : le coefficient de compressibilité plastique à l'état saturé (coefficient $\lambda$ du modèle CAM CLAY MODIFIE) $r$ : Constante liée à la rigidité du sol quand la succion tend vers l'infini $\left(r=\frac{\lambda(s \rightarrow \infty)}{\lambda(0)}, \mathrm{r}<1\right)$

$\beta$ : Un paramètre qui contrôle la vitesse d'augmentation de la rigidité avec la succion.

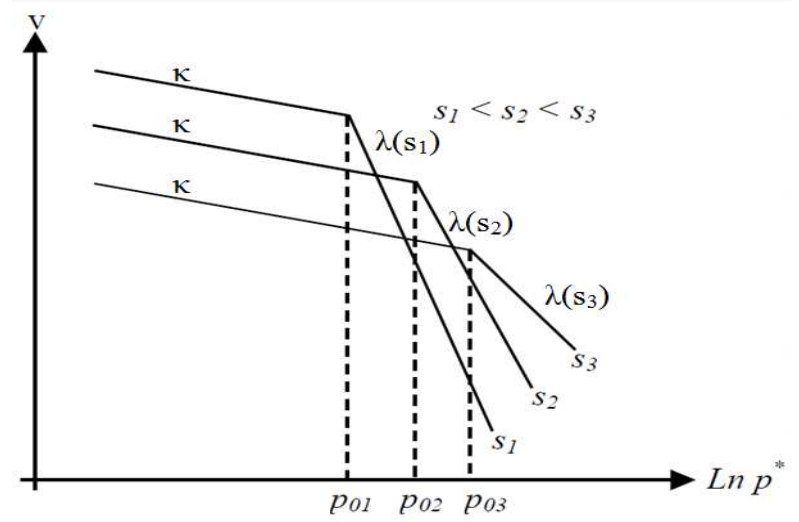

Fig.1. Réponse volumique lors d'un essai de compression à succion contrôlée [4]

La pression de pré-consolidation $p_{0 s}$ [figure 1] représente la limite d'élasticité du sol pour une succion donnée. Elle limite la zone où le comportement du sol est élastique (zone sur-consolidée de pente $\kappa$ ) de la zone des déformations irréversibles (zone normalement consolidée de pente $\lambda(\mathrm{s}))$.

Cette pression de pré-consolidation augmente avec la succion. La dite augmentation peut être représentée dans le plan $\left(s-p^{*}\right)$ par la courbe LC (Loading Collapse) [figure 2]. La courbe LC est, alors, une surface de charge puisqu'elle représente la limite entre la zone élastique et 
la zone de déformations plastiques. Elle est formulée comme suit :

LC: $\quad \frac{p_{0}}{p_{c}}=\left[\frac{p_{0}^{*}}{p_{c}}\right]^{\frac{\lambda(0)-\kappa}{\lambda(s)-\kappa}}$

Avec:

$p_{0}$ : la pression de pré-consolidation apparente sous la succion $\mathrm{s}$;

$p_{c}$ : une pression de référence [5]

$p_{0} *$ : la pression de pré-consolidation à l'état saturé ;

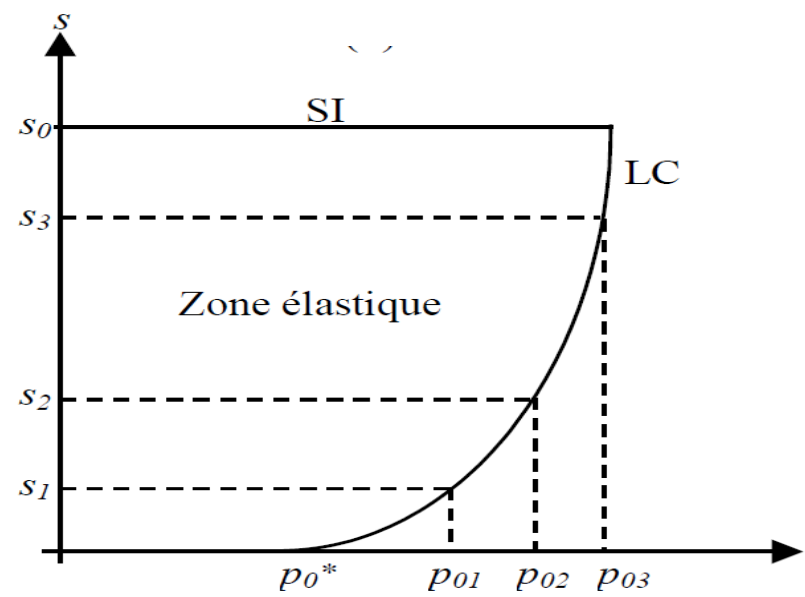

Fig.2. Définition de la surface de charge LC dans le plan $\left(\mathrm{p}^{*}-\mathrm{s}\right)[4]$

\subsection{Surfaces de charge «SI » et «SD » dans le plan $\left(p^{*}, \mathrm{~s}\right)$}

Un gonflement de la microstructure par humidification (diminution de la succion) peut engendrer une augmentation irréversible de l'indice des vides macrostructural $\mathrm{e}_{\mathrm{M}}$, et inversement, un rétrécissement de la microstructure par dessiccation (augmentation de la succion) peut induire une diminution irréversible de $\mathrm{e}_{\mathrm{M}}$. La limite entre la zone élastique et celle des déformations irréversibles de la macrostructure est définie par deux ligne parallèles à NL «neutral line ». les deux lignes, représentant des surfaces de charges relatives à la succion, sont notées: SI (suction increase) et SD (suction decrease) [figure 3].

Il est à préciser que la ligne neutre NL est orientée à $45^{\circ}$ par rapport aux axes et sépare la zone du gonflement microstructural de celle du retrait microstructural.

Les surfaces de charge SD et SI sont exprimés comme suit :

$S D: p^{*}+s-s_{h}=0$
$S I: p^{*}+s-s_{0}=0$
$\mathrm{S}_{\mathrm{h}}$ : est la succion à partir de laquelle les déformations plastiques apparaissent lors de l'humidification à contrainte moyenne nette nulle.

$\mathrm{S}_{0}$ : est la succion à partir de laquelle les déformations plastiques apparaissent lors de la dessiccation à contrainte moyenne nette nulle.

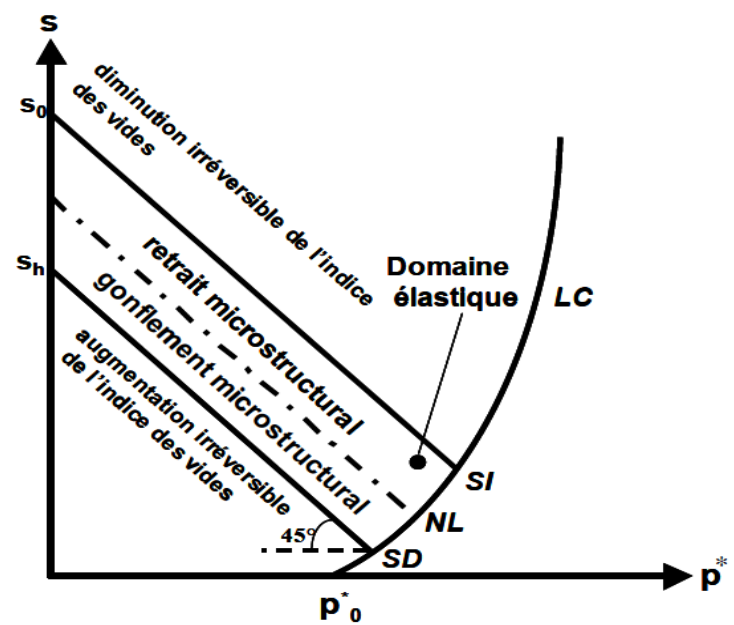

Fig.3. Surfaces de charge « SI » et « SD » du modèle $\mathrm{BE} \times \mathrm{M}[\underline{3}]$

\subsection{Prise en compte des contraintes déviatoriques}

Dans le cas d'un déviateur q, la surface de charge globale pourrait être décrite, dans le plan ( $\left.\mathrm{p}^{*}, \mathrm{q}, \mathrm{s}\right)$, par une succession d'ellipses, suivant l'axe des succions, inclinés de $45^{\circ}$ par rapport aux deux axes $\mathrm{p}^{*}$ et $\mathrm{s}$ [ $\underline{\text { figure }}$ 4]. Les projections de ces surfaces de charge dans le plan $\left(p^{*}, \mathrm{~s}\right)$ et $\left(p^{*}, \mathrm{q}\right)$ sont représentées dans [figures 5]. La méthode de détermination de l'équation de cette surface de charge fait l'objet du paragraphe suivant.

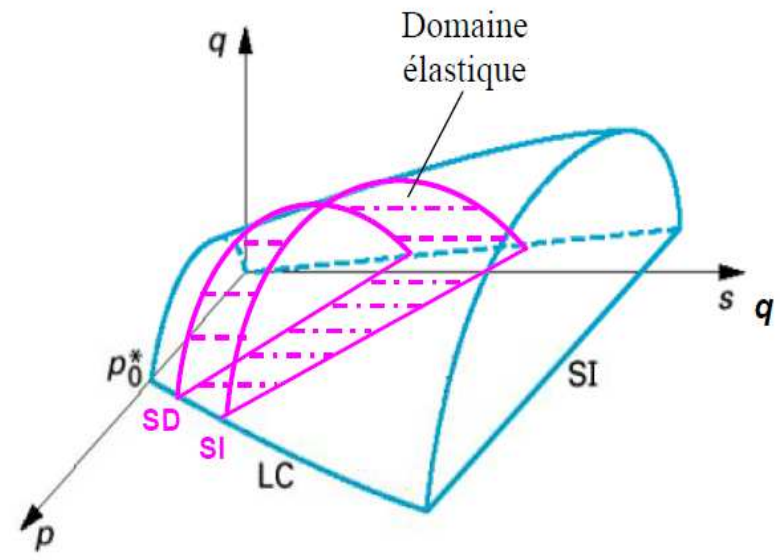

Fig.4. représentation tridimentionnelle des surfaces de charge dans l'espace $\left(\mathrm{p}^{*}, \mathrm{q}, \mathrm{s}\right)$ [6]

avec: 


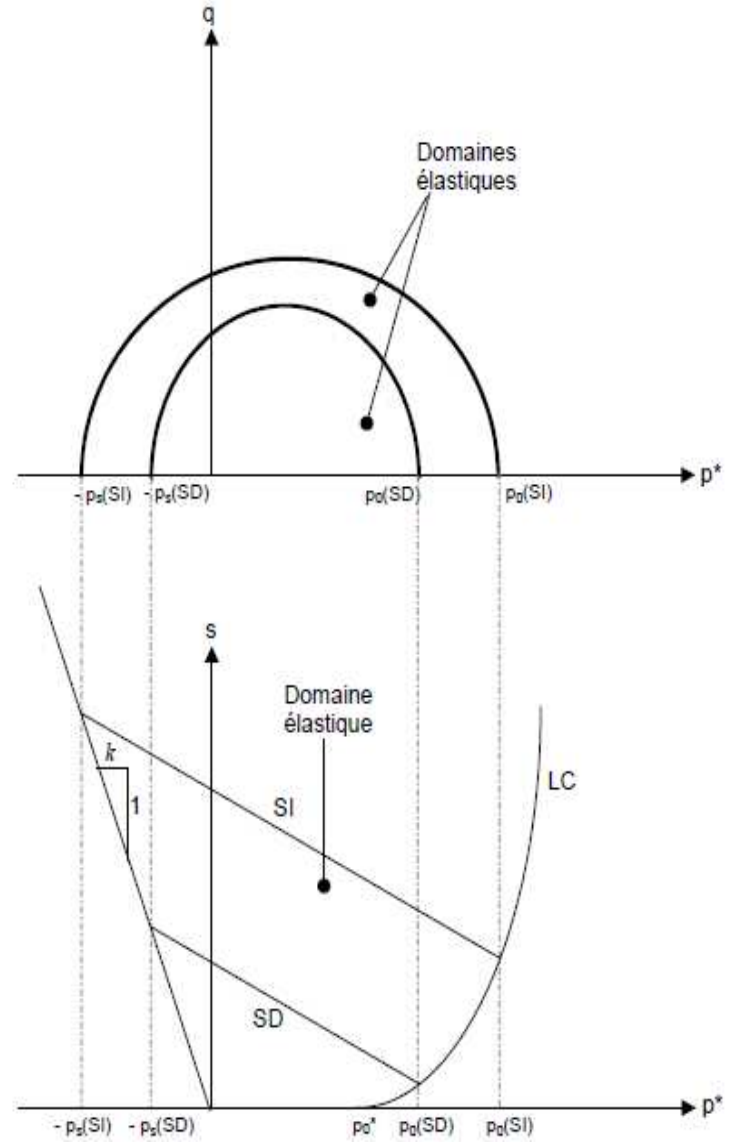

Fig.5. projection des surfaces de charge dans les plans $\left(p^{*}, q\right)$ et $\left(p^{*}, s\right)[6]$

\section{Détermination de la surface de charge globale « $F$ »}

La fonction de charge « $\mathrm{F}$ » est l'équation de la surface de charge globale en tenant compte de l'ensemble des surfaces de charge mises en jeu. C'est une fonction scalaire de la contrainte $\underline{\underline{\sigma}}$ et de la succion $s$ dont la détermination est difficile mais primordiale pour résoudre le problème élastoplastique du sol gonflant. Vu la complexité du problème, il est plus pratique de calculer l'équation d'une ellipse pour chaque état de contrainte donnée [6].

Soit le sol à un état de contrainte donnée $\left(p_{A}^{*}, s_{A}, \mathrm{q}_{A}\right)$ où $\mathbf{A}$ est le point qui correspond à cet état de contrainte dans le plan $\left(p^{*}, s, \mathrm{q}\right)$ et soit $\mathbf{A}^{\prime}$ la projection de $\mathbf{A}$ dans le plan $\left(p^{*}, s\right)$ [figure 6]. La droite passant par le point $\mathbf{A}^{\prime}$ et parallèle à SI et SD coupe la droite de cohésion au point $\mathbf{E}$ et la courbe $\mathrm{LC}$ au point $\mathbf{F}$. La fonction de charge recherchée $« \mathrm{~F} »$ est donc une ellipse de grand axe $I=\|E F\|$ et de petit axe $J=\|O M\|$ dans l'espace $\left(p^{*}-s-\mathrm{q}\right)\left(O\left(p_{O}^{*}, s_{O}\right)\right.$ étant le milieu de $\left.[\mathrm{EF}]\right)$ et la droite (EF) a pour équation: $p^{*}+s-b=0$ où $b=p^{*}\left(A^{\prime}\right)+s\left(A^{\prime}\right)$

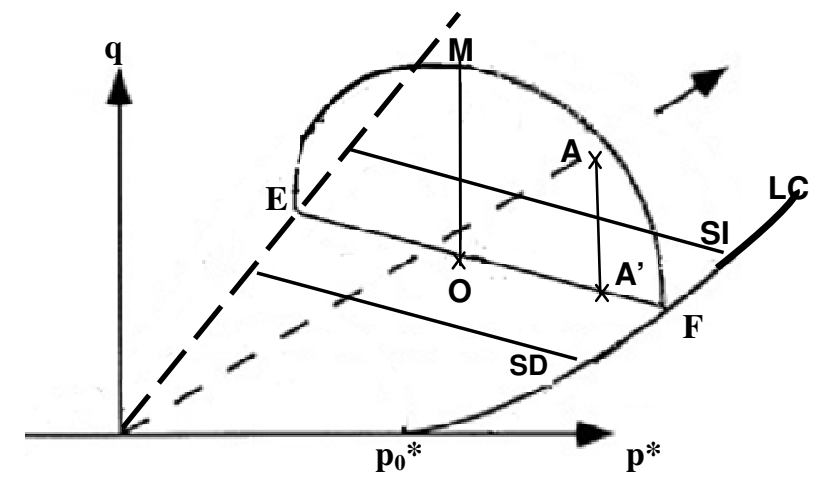

Fig.6. Projection de l'état de contrainte dans le plan $\left(\mathrm{p}^{*}, \mathrm{~s}\right)$ et l'ellipse représentant la fonction de charge

La détermination de la longueur I nécessite le calcul $\mathrm{du}$ point d'intersection de la droite (EF) avec la surface de charge LC (point F) et du point d'intersection de (EF) avec la droite de cohésion (point E). Pour ce faire, nous sommes remmenés à résoudre les systèmes d'équations à deux inconnus suivantes :

Point $\mathrm{E}:\left\{\begin{array}{l}p^{*}+s-b=0 \\ p^{*}=-k s\end{array}\right.$

Point F : $\left\{\begin{array}{l}p^{*}+s-b=0 \\ p^{*}=p_{c}\left[\frac{p_{0}^{*}}{p_{c}}\right]^{\frac{\lambda(0)-\kappa}{\lambda(s)-\kappa}}\end{array}\right.$

L'équation (5) relative au point $E\left(p_{E}^{*}, s_{E}\right)$ est facile à résoudre et les coordonnées du point $\mathrm{E}$ peuvent être données comme suit :

$$
E:\left\{\begin{array}{l}
p_{E}^{*}=\frac{b k}{k-1} \\
s_{E}=\frac{b}{1-k}
\end{array}\right.
$$

La détermination des coordonnées du point $F\left(p_{F}^{*}, s_{F}\right)$ revient à résoudre l'équation $f\left(s_{F}\right)=0$ suivante :

$p_{c}\left[\frac{p_{0}^{*}}{p_{c}}\right] \frac{\lambda(0)-\kappa}{\lambda(0)\left[(1-r) e^{\left.-\beta . \mathrm{s}_{F}+r\right]-\kappa}\right.}+s_{F}-b=0$

ou $b=p^{*}\left(A^{\prime}\right)+s\left(A^{\prime}\right)$

et $p_{0}^{*}, p_{c}, \lambda(0), \kappa, r$ et $\beta$ sont des paramètres données du modèle $\mathrm{BE} \times \mathrm{M}$. 
si l'on pose :

$$
\begin{aligned}
& C_{1}=p_{c} \\
& C_{2}=\frac{p_{0}^{*}}{p_{c}} \\
& C_{3}=\lambda(0)-\kappa \\
& C_{4}=\lambda(0)(1-r) \\
& C_{5}=\lambda(0) r-\kappa
\end{aligned}
$$

l'équation $f\left(s_{F}\right)=0$ devient :

$$
C_{1} C_{2} \frac{C_{3}}{C_{4} e^{-\beta s_{F}}+C_{5}}+s_{F}-b=0
$$

Ce système est insolvable analytiquement. nous sommes donc ramenés à le résoudre numériquement en utilisant la méthode de Newton pour des valeurs données des paramètres $p_{0}^{*}, p_{c}, \lambda(0), \kappa, r$ et $\beta$ et pour un état de contrainte donné.

La résolution numérique par la méthode de Newton consiste à donner une solution de départ $\left(s_{A^{\prime}}\right.$ par exemple) et faire des itérations ;

$s_{F}^{k+1}=s_{F}^{k}-\frac{f\left(s_{F}^{k}\right)}{\frac{\partial f}{\partial s}\left(s_{F}^{k}\right)}$

où :

$s_{F}^{k+1}$ et $s_{F}^{k}$ : les solution de l'équation aux rangs $k+1$ et $k$ respectivement

$\frac{\partial f}{\partial s}\left(s_{F}^{k}\right)$ :la dérivée de la fonction $f$ par rapport à la succion donnée par :

$\frac{\partial f}{\partial s}\left(s_{F}^{k}\right)=\frac{C_{1} C_{2} \frac{C_{3}}{C_{4} e^{-\beta s_{F}^{k}}+C_{5}} C_{3} C_{4} \beta e^{-\beta s_{F}^{k}} \log C_{2}}{\left(C_{4} e^{-\beta s_{F}^{k}}+C_{5}\right)^{2}}+1$

Il convient de tester la convergence après chaque itération en vérifiant que l'écart entre les valeurs de succion retrouvées aux rang $\mathrm{k}$ et $\mathrm{k}+1$ est inférieur à la tolérance fixée par l'utilisateur. Finalement :

$\mathrm{F}:\left\{\begin{array}{l}p_{F}^{*}=b-s_{F}^{k} \\ s_{F}=s_{F}^{k}\end{array}\right.$

Cette méthode de résolution converge vers la solution exacte quelle que soit la solution de départ donnée.

Une fois les coordonnées de E et F (équations (7) et (8)) sont calculées, la longueur du grand axe I est donnée par la formule suivante :

$$
I=\|\mathrm{EF}\|=\sqrt{\left(p_{F}^{*}-p_{E}^{*}\right)^{2}-\left(s_{F}-s_{E}\right)^{2}}
$$

La longueur du petit axe $J=\|O M\|$ ne pourrait pas être déterminée directement vu qu'elle représente la distance du point $\mathrm{O}$ (milieu de $[\mathrm{EF}])$ à l'ellipse dont le but final de cet article est de chercher son l'équation! Mais en observant que cette distance est égale à celle du point $\mathrm{O}$ à l'ellipse de grand axe [E'F'] [figure 7] dont l'équation est connue (Modèle de Barcelone «Barcelona Basic Model $B B M \gg$ décrit par Alonso et al. [7]:

$$
q^{2}-M^{2}\left(p^{*}+p_{s}\right)\left(p_{0}(s)-p^{*}\right)=0
$$

où :

M: est la pente de la ligne d'état critique CSL dans le plan $\left(p^{*}, q\right)$ supposée indépendante du chemin de chargement et de la succion []].

$\mathrm{P}_{0}(\mathrm{~s})$ : est la pression de préconsolidation à succion constante donnée par l'équation (2) ;

$\mathrm{P}_{\mathrm{s}}$ : donnée par $p_{s}=k . s$, avec $\mathrm{k}$ un paramétre désignant l'augmentation de la cohésion avec la succion [9]. $p_{s}=k . s$ est appelée droite de cohésion.

on aura :

$$
J=2 q=2 M \sqrt{\left(p_{O}^{*}+p_{S}\left(s_{O}\right)\right)\left(p_{0}\left(s_{O}\right)-p_{O}^{*}\right)}
$$

ou $p_{O}^{*}$ et $s_{O}$ sont les coordonnées du point $\mathrm{O}$ : $\mathrm{O}:\left\{\begin{array}{l}p_{O}^{*}=\frac{p_{F}^{*}+p_{E}^{*}}{2} \\ s_{O}=\frac{s_{F}^{*}+s_{E}^{*}}{2}\end{array}\right.$

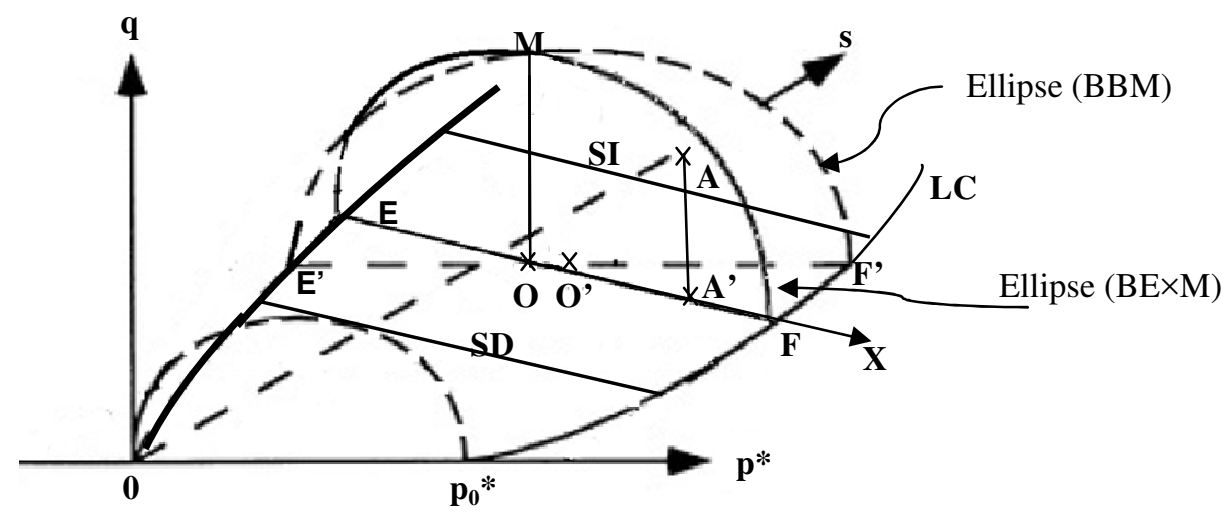

Fig.7. détermination du petit axe de l'ellipse « $\mathrm{F} »$ du modèle $\mathrm{BE} \times \mathrm{M}$ en utilisant le modèle $\mathrm{BBM}$ 
$p_{s}\left(s_{O}\right)=k \cdot s_{O}$

$p_{0}\left(s_{O}\right)=p_{c}\left[\frac{p_{0}^{*}}{p_{c}}\right]^{\frac{\lambda(0)-\kappa}{\lambda(0)\left[(1-r) e^{-\beta s_{O}}+r\right]-\kappa}}$

l'équation cartésienne de l'ellipse dans le plan local $(\mathrm{X}, \mathrm{q})$ est alors:

$\left[\frac{X}{(I / 2)}\right]^{2}+\left[\frac{q}{(J / 2)}\right]^{2}=1$

qui devient, après transformation des coordonnées, dans le plan $\left(p^{*}, q\right)$ :

$F=-1+\frac{2\left(p_{O}^{*}\right)^{2}}{\left(\frac{I}{2}\right)^{2}}-\frac{4 p_{O}^{*}}{\left(\frac{I}{2}\right)^{2}} p^{*}+\frac{2}{\left(\frac{I}{2}\right)^{2}} p^{* 2}+\frac{q^{2}}{\left(\frac{J}{2}\right)^{2}}$

en posant:

$C_{F 1}=-1+\frac{2\left(p_{o}^{*}\right)^{2}}{\left(\frac{I}{2}\right)^{2}}$

$C_{F 2}=-\frac{4 p_{o}^{*}}{\left(\frac{I}{2}\right)^{2}}$

$C_{F 3}=\frac{2}{\left(\frac{I}{2}\right)^{2}}$

$C_{F 4}=\frac{1}{\left(\frac{J}{2}\right)^{2}}$

on peut écrire: $F=C_{F 1}+C_{F 2} p^{*}+C_{F 3} p^{* 2}+C_{F 4} q^{2}$

\section{Détermination des dérivées de la surface de charge « $F$ »}

La dérivée de $\mathrm{F}$ par rapport au vecteur des contraintes pourrait être calculée comme suit :

$\frac{\partial F}{\partial \sigma}=\frac{\partial F}{\partial p^{*}} \frac{\partial p^{*}}{\partial \sigma}+\frac{\partial F}{\partial q} \cdot \frac{\partial q}{\partial \sigma}$

avec: $p^{*}=\frac{\sigma_{1}+\sigma_{2}+\sigma_{3}}{3}-u_{a}$ et $q=\sqrt{3 J_{2}}$

où $J_{2}$ étant le deuxième invariant du tenseur déviateur des contraintes. On peut écrire alors :

$\frac{\partial p^{*}}{\partial \sigma}=\left(\begin{array}{llllll}\frac{1}{3} & \frac{1}{3} & \frac{1}{3} & 0 & 0 & 0\end{array}\right)^{T}$

$$
\frac{\partial q}{\partial \sigma}=3\left(\begin{array}{c}
\frac{1}{2 q}\left(\sigma_{1}-\frac{\sigma_{1}+\sigma_{2}+\sigma_{3}}{3}\right) \\
\frac{1}{2 q}\left(\sigma_{2}-\frac{\sigma_{1}+\sigma_{2}+\sigma_{3}}{3}\right) \\
\frac{1}{2 q}\left(\sigma_{3}-\frac{\sigma_{1}+\sigma_{2}+\sigma_{3}}{3}\right) \\
\frac{1}{q} \sigma_{4} \\
\frac{1}{q} \sigma_{5} \\
\frac{1}{q} \sigma_{6}
\end{array}\right)
$$

et $\frac{\partial F}{\partial q}=\frac{2}{\left(\frac{J}{2}\right)^{2}} q$

pour obtenir finalement :

$\frac{\partial F}{\partial \sigma}=3\left(\begin{array}{c}\frac{4}{J^{2}}\left(\sigma_{1}-\frac{\sigma_{1}+\sigma_{2}+\sigma_{3}}{3}\right)+\frac{16}{3 I^{2}}\left(p^{*}-p_{O}^{*}\right) \\ \frac{4}{J^{2}}\left(\sigma_{2}-\frac{\sigma_{1}+\sigma_{2}+\sigma_{3}}{3}\right)+\frac{16}{3 I^{2}}\left(p^{*}-p_{O}^{*}\right) \\ \frac{4}{J^{2}}\left(\sigma_{3}-\frac{\sigma_{1}+\sigma_{2}+\sigma_{3}}{3}\right)+\frac{16}{3 I^{2}}\left(p^{*}-p_{O}^{*}\right) \\ \frac{8}{J^{2}} \sigma_{4} \\ \frac{8}{J^{2}} \sigma_{5} \\ \frac{8}{J^{2}} \sigma_{6}\end{array}\right)$

Quant à la dérivée de $\mathrm{F}$ par rapport à la succion $\frac{\partial F}{\partial s}$, elle doit être calculée numériquement (méthode de différence finie centrée) vu que la fonction de charge $F$ ne dépend pas explicitement de la succion. Ainsi, et pour un état de contrainte donnée :

$\frac{\partial F}{\partial s}=\frac{F(s+\Delta s)-F(s-\Delta s)}{2 \Delta s}$

Le modèle $\mathrm{BE} \times \mathrm{M}$ repose sur trois paramètres d'écrouissage $; p_{0}^{*}, s_{0}$ et $s_{h}$, mais seul le paramètre $p_{0}^{*}$ qui intervient dans l'équation de la fonction de charge $\mathrm{F}$. Ainsi, la dérivée $\frac{\partial F}{\partial \chi}$ n'est autre que $\frac{\partial F}{\partial p_{0}^{*}}$. Cette dernière peut être calculée numériquement aussi comme ainsi : 


$$
\frac{\partial F}{\partial p_{0}^{*}}=\frac{F\left(p_{0}^{*}+\Delta p_{0}^{*}\right)-F\left(p_{0}^{*}-\Delta p_{0}^{*}\right)}{2 \Delta p_{0}^{*}}
$$

\section{Conclusion}

La détermination de la fonction de charge $« F » d u$ modèle $\mathrm{BE} \times \mathrm{M}$ et de ses dérivées est une étape primordiale pour résoudre le problème élastoplastique d'un sol gonflant non saturé. En effet, Ceci permettra de déterminer les contraintes initiales, traiter l'intégrale volumique correspondante et finalement permettre l'implémentation du modèle $\mathrm{BE} \times \mathrm{M}$ dans un code de calcul basé sur la méthode des éléments de frontière.

\section{Références}

1. J.El Brahmi, M. Zoukaghe, Numerical Analysis of Nonlinear Coupled Problems, Sustainable Civil Infrastructures, 262-274 (2017)

2. A.Gens and E.E. Alonso. A framework for the behaviour of unsaturated expansive clays. Canadian Geotechnical Journal, 29, 1013-1032 (1992)

3. E.E. Alonso, J.Vaunat and A.Gens, Modelling the mechanical behaviour of expansive clays. Engineering Geology, 54, 173-183 (1999).

4. E.E. Alonso, A.Gens and D.W. Hight. General report - Special problem soils. Proceedings of the 9th European Conference on Soil Mechanics and Foundation Engineering, Dublin, 3, 1087-1146 (1987).

5. S.J.Wheeler, D.Gallipolo and M.Karstunen, Comments on use of the Barcelona Basic Model for unsaturated soils. International Journal for Numerical and Analytical Methods in Geomechanics, 26,15611571 (2002)

6. M.Mrad, Modélisation du comportement hydromécanique des sols gonflants non saturés. Thèse de doctorat, Institut national polytechnique de Loraine, Ecole Nationale Supérieure de Géologie de Nancy (2000)

7. E.E, Alonso, A.Gens, A.Josa, A constitutive model for partially saturated soils. Géotechnique, 40, $\mathrm{n}^{\circ} 3$, 405-430 (1990)

8. S.J,Wheeler V.and Sivakumar. An elasto-plastic critical framework for unsaturated soil. Géotechnique, 45, ${ }^{\circ} 1,35-53$ (1995)

9. L.Miao, S.Lius and Y.Lai. Research of soil-water characteristics and shear strength features of Nanyang expansive soil, Engineering Geology, 65, 261-267 (2002) 